\title{
The Crystal Structure of Silk Fibroin
}

\author{
BY J. O. WARWICKER \\ British Cotton Industry Research Association, Shirley Institute, Didsbury, Manchester 20, England
}

(Received 16 November 1953 and in revised form 1 April 1954)

\begin{abstract}
An X-ray investigation of a precipitate obtained by the action of pancreatic enzymes on an aqueous solution of silk fibroin suggests that the precipitate has essentially the same crystal structure as the silk fibre. Since the precipitate consists almost entirely of glycine, alanine and serine residues, it is concluded that only one crystalline phase, composed of these three aminoacids, is present in silk fibroin.

The precipitate, which is more highly crystalline than silk fibroin, has a density of 1.40 g.cm..$^{-3}$. This value is taken as an approximation to the density of the crystalline regions of the silk fibre.

From photographs obtained when silk fibres are oscillated in the $\mathrm{X}$-ray beam, it is concluded that the fibroin structure possesses a twofold screw axis.

Consideration of all the available evidence suggests an orthorhombic unit cell and the space group $P 22_{1} 2_{1}$ for silk fibroin. A detailed structure is proposed, and it is shown that intensities of $\mathrm{X}$-ray reflexions calculated from it are in reasonable agreement with those observed.
\end{abstract}

\section{Introduction}

The diffraction of X-rays by silk, first observed by Herzog \& Jancke (1920), has been frequently used as a means of investigating the structure of silk fibroin. Brill (1923) studied silks of various types, and concluded that they all had essentially the same orthorhombic crystal lattice and that the only amino-acids that could be present in the crystalline component were glycine and alanine. Meyer \& Mark (1928), who were the first to interpret the crystal structure of silk in terms of long polypeptide chains, suggested the possibility that the crystallites might have a monoclinic, instead of an orthorhombic, unit cell; the space groups assigned to the two forms were $P 2$ and $P 222$ respectively. The view that the crystalline regions were built up from glycine and alanine residues was supported by the work of Goldschmidt \& Strauss (1930), who, by oxidizing silk with alkaline hypobromite, obtained a resistant fraction which contained only glycine and alanine but gave the same X-ray diagram as the unoxidized silk. Meyer, Fuld \& Klemm (1940) later made the suggestion that serine units might take the place of some of the alanine units in the crystalline regions. A further $\mathrm{X}$-ray investigation of silks of various types was carried out by Kratky \& Kuriyama (1931), who made use of highly oriented films of fibroin prepared from the glands of the silkworm (Kratky, 1929). These workers showed that the X-ray diagram given by silk of the type Bombyx mori could be accounted for by eleven different monoclinic unit cells, but, by rejecting those which gave obviously improbable values for the density of the crystalline regions, they were able to reduce this number to six. Brill (1942) drew attention to the similarity between the X-ray diagrams of silk fibroin and synthetic polyamides, and, on the basis of analogy with structures suggested for the latter, he proposed both a unit cell and a structure for silk. The monoclinic unit cell which he adopted is closely related to two of those deduced by Kratky \& Kuriyama.

It is assumed in all the work reviewed above that silk fibroin contains only one crystalline phase. The suggestion that there might be two crystalline phases was first put forward by Trogus \& Hess (1933) as a possible explanation of their observation that certain $\mathrm{X}$-ray reflexions were the same for all silks whereas others varied with the type of silk. Recently an interpretation of the X-ray diagram of silk fibroin in terms of two crystalline phases has been advanced by Happey \& Hyde (1952), who suggest that one of the phases is composed of glycine and alanine residues, with some replacement of alanine by serine, and the other is associated with tyrosine residues.

The foregoing review shows that no general agreement has yet been reached about the unit cell of the fibroin lattice, much less about the details of the structure, and it is clear that the evidence hitherto available is insufficient for a solution of the problem. The present paper gives some new experimental evidence bearing upon the structure of silk of the type Bombyx mori. Part of this evidence is derived from the study of a fraction obtained by the action of pancreatic enzymes on silk fibroin; the remainder comes from new X-ray data obtained with fibrous silk. On the basis of this new information and current views about the configuration of the polypeptide chain, a unit cell and space group are assigned to the fibroin lattice and a possible arrangement of the polypeptide chains is suggested.

\section{$\mathrm{X}$-ray diagrams of silk fibroin and a fraction obtained from it}

In an investigation made in these laboratories (Drucker \& Smith, 1950; Drucker, Hainsworth \& Smith, 
Table 1. Comparison of $X$-ray data for silk fibroin, regenerated fibroin, and the insoluble fraction

Powder diagrams

\begin{tabular}{|c|c|c|c|c|c|c|c|c|c|c|c|c|}
\hline \multirow[b]{2}{*}{ Ring } & \multicolumn{3}{|c|}{ Fibrous silk } & \multicolumn{3}{|c|}{ Regenerated fibroin } & \multicolumn{3}{|c|}{ Insoluble fraction } & \multicolumn{3}{|c|}{ Fibre diagram; silk } \\
\hline & $\operatorname{Sin} \theta$ & $d(\AA)$ & Intensity & $\operatorname{Sin} \theta$ & $d(\AA)$ & Intensity & $\operatorname{Sin} \theta$ & $d(\AA)$ & Intensity & Spot & $\operatorname{Sin} \theta$ & Intensity \\
\hline $\begin{array}{l}R_{1} \\
R_{2}\end{array}$ & \multicolumn{3}{|c|}{$\begin{array}{l}\text { Diffuse haze } \\
\text { Do }\end{array}$} & $0 \cdot 083$ & Diffuse haze & $\begin{array}{l}m ; \text { diffuse } \\
\text { haze }\end{array}$ & $\begin{array}{l}0 \cdot 085 \\
0 \cdot 165\end{array}$ & $\begin{array}{l}9 \cdot 00 \\
4 \cdot 67\end{array}$ & $\begin{array}{l}s \\
v s\end{array}$ & $\begin{array}{l}A_{1} \\
A_{2}\end{array}$ & $\begin{array}{l}0 \cdot 083 \\
0 \cdot 164\end{array}$ & $\begin{array}{c}s \\
s ; \text { diffuse }\end{array}$ \\
\hline$R_{3}$ & $0 \cdot 178$ & $4 \cdot 33$ & $v s$ & $0 \cdot 178$ & $4 \cdot 33$ & vs & $0 \cdot 181$ & $4 \cdot 26$ & $v v s$ & $A_{3}$ & $0 \cdot 179$ & vvs \\
\hline$R_{4}$ & $0 \cdot 215$ & $3 \cdot 59$ & $s$ & 0.215 & 3.59 & $\boldsymbol{s}$ & 0.213 & $3 \cdot 61$ & $\boldsymbol{s}$ & & $\begin{array}{l}0 \cdot 218 \\
0 \cdot 208\end{array}$ & $\begin{array}{l}m \\
v s\end{array}$ \\
\hline $\begin{array}{l}R_{\bar{s}} \\
R_{\mathrm{g}}\end{array}$ & \multicolumn{3}{|c|}{ Not observed } & \multicolumn{3}{|c|}{ Not observed } & \multicolumn{3}{|c|}{ Not observed } & $\mathrm{II}_{1}$ & $\begin{array}{l}0.234 \\
0.252\end{array}$ & 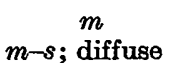 \\
\hline$R_{7}$ & $0 \cdot 280$ & $2 \cdot 76$ & $m$ & $0 \cdot 280$ & $2 \cdot 76$ & $m$; diffuse & 0.278 & $2 \cdot 77$ & $s$ & $\mathrm{II}_{2}^{\prime}$ & $\begin{array}{l}0 \cdot 272 \\
0 \cdot 286\end{array}$ & $\begin{array}{l}w \\
m\end{array}$ \\
\hline$R_{8}$ & \multicolumn{3}{|c|}{ Not observed } & \multicolumn{3}{|c|}{ Not observed } & 0.330 & $2 \cdot 34$ & $w$ & $\mathrm{I}_{4}, \mathrm{II}_{A_{5}}$ & $\begin{array}{c}0.335,0 \cdot 333 \\
0 \cdot 324\end{array}$ & $\begin{array}{l}m m \\
m\end{array}$ \\
\hline$R_{9}$ & $0 \cdot 342$ & $2 \cdot 25$ & $m$ & $\mathbf{0 \cdot 3 4 0}$ & $2 \cdot 26$ & $m$; diffuse & $0 \cdot 342$ & $2 \cdot 25$ & $m$ & $\operatorname{III}_{1}$ & 0.339 & $m$ \\
\hline$R_{10}$ & $0 \cdot 379$ & $2 \cdot 04$ & $\boldsymbol{8}$ & 0.379 & $2 \cdot 04$ & $s$; diffuse & $0 \cdot 379$ & $2 \cdot 04$ & $s$ & $\mathrm{III}_{2}$ & $0 \cdot 367$ & $m$ \\
\hline$R_{11}^{10}$ & 0.418 & $1 \cdot 84$ & $w$ & 0.418 & $1 \cdot 84$ & $v w$ & 0.418 & $1 \cdot 84$ & $w$ & $\mathrm{III}_{3}$ & 0.423 & $\boldsymbol{w}$ \\
\hline$R_{12}$ & 0.479 & $1 \cdot 61$ & verw & \multicolumn{3}{|c|}{ Not observed } & 0.479 & $\mathbf{1 \cdot 6 1}$ & $v v w$ & $I V_{1}$ & $0 \cdot 464$ & $w$ \\
\hline$R_{13}$ & 0.499 & $1 \cdot 54$ & $w$; diffuse & 0.494 & 1.56 & $w$ & 0.499 & 1.54 & $w-m$ & $A_{6}$ & $0 \cdot 495$ & $w$ \\
\hline$R_{14}$ & 0.551 & $1 \cdot 40$ & vow & \multicolumn{3}{|c|}{ Not observed } & 0.551 & $1 \cdot 40$ & $v v w$ & & 0.551 & $m$ \\
\hline$R_{15}$ & 0.595 & $1 \cdot 29$ & $w$ & 0.585 & $1 \cdot 32$ & $w$ & 0.595 & $1 \cdot 29$ & $w$ & $\operatorname{III}_{4}$ & 0.597 & vo \\
\hline & 0.669 & $1 \cdot 15$ & $m$ & $0 \cdot 669$ & $1 \cdot 15$ & $m$ & $0 \cdot 669$ & $1 \cdot 15$ & $m$ & $\mathrm{VI}_{0}$ & 0.656 & $s$ \\
\hline
\end{tabular}

$1953 a, b)$ it has been shown that the action of pancreatic enzymes on silk fibroin in aqueous solution leads to the formation of a precipitate consisting. mainly of glycine, alanine and serine residues, with only a small proportion of tyrosine residues. This composition suggested that the precipitate might be similar in structure to the crystalline regions in silk, and a comparison of the X-ray diagrams of silk fibroin and the precipitate was therefore made. For this purpose it was necessary to use powder diagrams. Silk fibroin was examined in two forms, viz. silk fibres cut to as short a length as possible, and the powdery material obtained by the regeneration of fibroin from its aqueous solution by the addition of ethyl alcohol. The X-ray photographs are shown in Fig. 1, and the data obtained from them, together with data from the fibre diagram of silk, are recorded in Table 1.

Inspection of the photographs and reference to the table show that the diagrams given by the silk and the regenerated fibroin are essentially the same. The diagram given by the precipitate bears a strong general resemblance to those obtained with fibroin, but is considerably sharper. However, a closer comparison reveals other differences: the diffraction rings $R_{2}, R_{6}$, and $R_{8}$ which are given by the precipitate do not appear in the fibroin diagram, and the ring $R_{5}$ is given by the fibroin but not by the precipitate. It will be shown that these differences are not inconsistent with the views that the precipitate has essentially the same crystal structure as fibroin, but before this question is discussed it is necessary to consider some features of the fibre diagram of silk and the probable mechanism of the formation of the precipitate.

In the $\mathrm{X}$-ray fibre diagram given by silk the reflexions fall into two distinct groups, viz. sharp and diffuse. The diffuse reflexions probably arise from distortion of the lattice, caused either by imperfect packing of side-chains within the lattice or by the presence of bulky side-chains on the fringes of the crystalline regions. According to this view, the planes which give rise to the diffuse reflexions might be expected to lie at right angles to the direction of the weakest bonds in the structure.

It is assumed (Drucker et al., 1953a,b) that in the attack on fibroin by the pancreatic enzymes the peptide chains are severed in such a way as to produce chain molecules consisting only of glycine, alanine and serine units except for a tyrosine unit at one end, and that these shortened chain molecules, containing no bulky side-chains except that of the terminal tyrosine unit, are able to crystallize from the solution. In these circumstances it would be expected that the packing of the chains would be more nearly perfect than in the original fibre, and that the resulting precipitate would therefore contain larger crystallites and a smaller proportion of amorphous material than the original silk. The effect of the recrystallization following attack by pancreatic enzymes should thus be to sharpen all the $\mathrm{X}$-ray reflexions, particularly those that were diffuse in the fibroin diagram. Further, the reflexions that were originally diffuse should tend to occur at slightly higher Bragg angles with the precipitate than with fibroin because of the smaller interplanar spacings which result from the improved packing of the polypeptide chains in the precipitate.

The fulfilment of these expectations is most clearly seen in connection with the diffraction ring $R_{1}$. This reflexion, subsequently indexed as 100 , is seen to be sharper, and the corresponding interplanar spacing is found to be slightly less, with the precipitate than with 

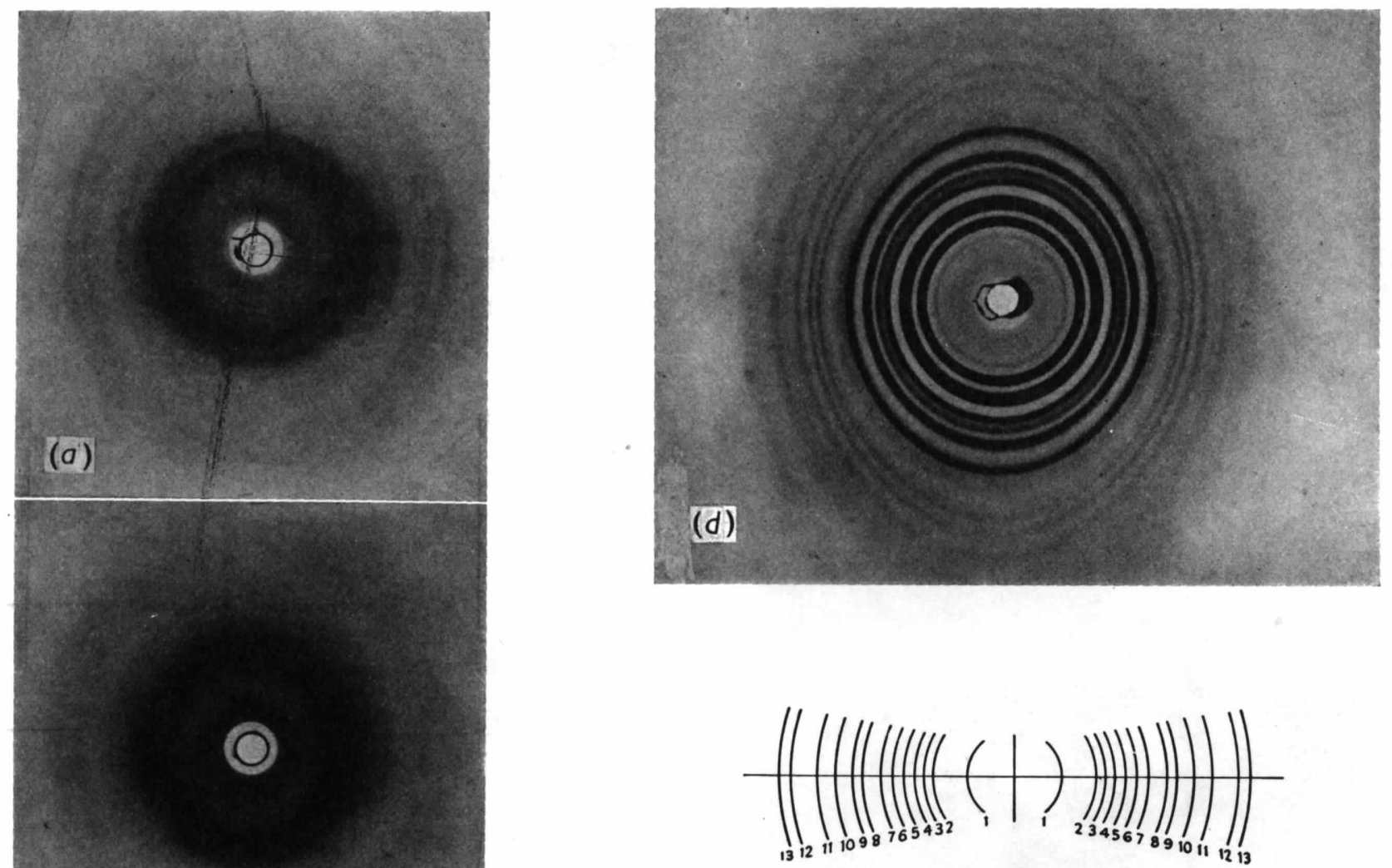

(e)

(b)

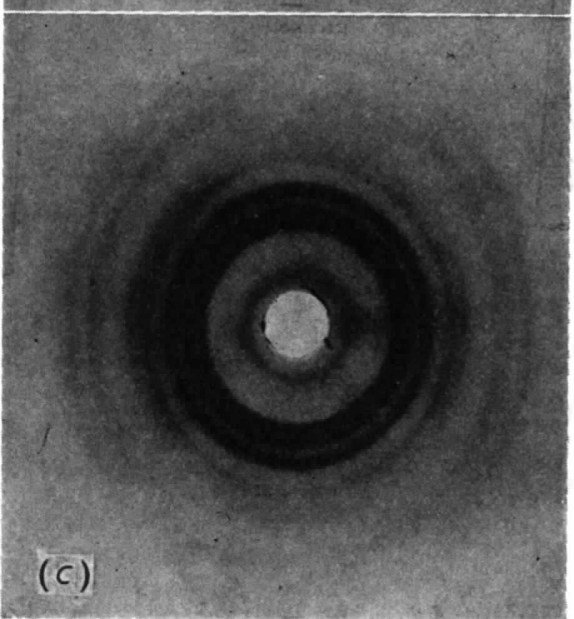

Fig. 1. Powder photographs of $(a)$ silk, $(b)$ regenerated fibroin, $(c)$ precipitate obtained by the action of pancreatic enzymes on aqueous solution of fibroin, and $(d)$ DL-alanyl-glycine. $(e)$ Key to $(a)$, $(b)$ and $(c)$. 
fibroin. This observation suggests that the main distortion of the fibroin lattice is perpendicular to the (100) planes, and it would therefore be expected that higher-order reflexions from this set of planes, as well as reflexions from closely connected planes, would show similar features. Thus the reflexions $A_{2}$ and $A_{4}$, indexed as 200 and 300 respectively, which are diffuse in the fibre diagram, are not distinguishable in the powder diagram of fibroin but do contribute to the powder diagram given by the precipitate. The composite spot $\left(A_{2}+A_{3}\right)$ shows as a single diffraction ring in the powder diagram of fibroin, but as a result of the recrystallization process it shows as two rings in the powder photograph given by the precipitate, the extra ring being $R_{2}$. Similarly, the ring $R_{5}$, which is visible in the powder diagram of fibroin only because of the overlapping of $\mathrm{II}_{1}$ and $A_{4}$, shifts to the position $R_{6}$ mainly because of sharpening of the reflexion corresponding to $A_{4}$. The absence of $R_{6}$ from the fibroin diagram and the absence of $R_{5}$ from the diagram given by the precipitate are thus explained. In a similar way, the presence of $R_{8}$ in the diagram from the precipitate can be explained as the result of the reinforcement of the $A_{5}$ reflexion by the sharpening of the reflexion $\mathrm{I}_{4}$ and $\mathrm{II}_{3}$ from the (401) and (302) planes respectively. Thus it is possible to account for all the differences between the powder diagrams given by fibroin and the precipitate, and it is concluded that the precipitate has the same crystal structure as silk, differing from it only in being more highly crystalline. That the precipitate has a high degree of crystallinity is shown by a comparison of its X-ray diagram with that given by the crystalline dipeptide DL-alanylglycine (Fig. 1).

The X-ray diagram given by the precipitate can also be used to test Happey \& Hyde's hypothesis that silk fibroin contains two crystalline phases. According to these workers, the first phase is composed of glycine and alanine units, with some replacement of alanine by serine, and has a monoclinic unit cell, whereas the second phase, which is associated with the tyrosine residues, has an orthorhombic unit cell. The reflexions in the fibre diagram of silk may be indexed on the basis of these two unit cells, with the results shown in Table 2. It should be noted that since $A_{1}$ and $A_{2}$ are the fundamental reflexions for the second phase, any alteration in this phase should result in a corresponding change in these reflexions. It is evident that $A_{1}$ is more clearly defined (as $R_{1}$ ) in the diagram from the precipitate than in the powder diagram from fibroin, in spite of the fact that the precipitate contains only about $1 \%$ of tyrosine, supposedly the most important constituent of the second phase. Moreover, the rings $R_{6}, R_{7}$ and $R_{10}$ would also have to be indexed as reflexions from the second phase, and they are all present in the diagram given by the precipitate. The evidence provided by the precipitate is thus strongly against the two-phase structure for silk suggested by Happey \& Hyde.

\section{Density and unit cell of fibroin crystallites}

It has already been mentioned that Kratky \& Kuriyama (1931) specified eleven different unit cells that were compatible with the results of their $\mathrm{X}$-ray examination of silk. Each of these unit cells is distinguished by a different density, and consequently a choice of the most probable unit cell for silk could be made if the actual density of the crystalline regions were known. If it is accepted that the precipitate described above has essentially the same structure as the crystalline portions of silk, then the density of this precipitate should provide a good approximation to that of the crystallites in the silk fibre. The density of the precipitate was found to be $1.398 \mathrm{~g} . \mathrm{cm} .^{-3}$. (According to Goodings \& Turl (1940), the density of silk is 1.353 g.cm. ${ }^{-3}$.) Since the precipitate is not perfectly crystalline, it may be presumed that the density of the crystallites in silk is slightly greater than 1.40 g.cm. ${ }^{-3}$.

Of the possible unit cells listed by Kratky \& Kuriyama, only the first gives a theoretical density in the required region, viz. 1.40 g.cm. ${ }^{-3}$; the modified form of this cell adopted by Brill gives a density of 1.375 g.cm..$^{-3}$. These values are derived on the assumption that the crystallites are built up from alternating glycine and alanine residues, and they will be increased if some of the alanine units are replaced by serine units. Smith and his collaborators give the molar ratios of glycine, alanine and serine in the precipitate as $3: 2: 1$, and if this is accepted as the composition of the crystallites in silk then the average weight of the repeating unit increases from 128, the value for a glycyl-alanyl unit, to 133; on this basis the theoretical density of Kratky \& Kuriyama's first cell becomes 1.459 g.cm. ${ }^{-3}$ and that of the Brill cell 1.429 g.cm. ${ }^{-3}$. The choice appears to lie between these two unit cells, and it will be shown later that a modified form of the Kratky-Kuriyama unit cell accords well with all the available evidence.

\section{Evidence from X-ray fibre photographs}

Evidence about the symmetry elements present in the fibroin structure may be obtained from a consideration of fibre diagrams given by silk. Fig. 2 shows the normal fibre photograph, taken with a stationary vertical bundle of fibres, and two photographs taken with a horizontal bundle which was oscillated through an angle of $15^{\circ}$ during the exposure.

The two oscillation photographs are of particular interest. The first of these (Fig. 2(b)), obtained when the fibre bundle was set at an angle of $15^{\circ}$ to the X-ray beam, clearly shows the 002 reflexion, but gives no indication of the presence of 001 or 003 reflexions. (The fibre axis is taken as the $c$ axis.) The second (Fig. 2(c)), for which the fibre bundle was set at an angle of $30^{\circ}$ to the beam, shows, in the original, the 004 and 006 reflexions, but is rather inconclusive with respect to the 005 reflexion; it is probable that what 
appears to be a weak 005 reflexion is caused by departures from the ideal arrangement in the side groups, leaving the main structure unchanged (cf. the interpretation by Legrand (1952) of the 030 reflexion in the cellulose $\mathrm{X}$-ray diagram). It may therefore be concluded that $00 l$ reflexions occur only when $l$ is even, and hence that the fibroin structure possesses a twofold screw axis.

The formation of a twofold screw axis from the polypeptide chains present in the crystalline portions of fibroin requires some discussion. In hydrolysates from fibroin Levy \& Slobodian (1952; Slobodian \& Levy, 1953) found the dipeptides alanyl-glycine and glycyl-alanine, but very little glycyl-glycine or alanylalanine, and they concluded that the sequence alanylglycyl-alanyl is an important one in this protein. If it is accepted that only glycine, alanine and serine residues occur in the crystalline regions of silk, then it seems probable that the repeating unit in the chain is glycyl-alanyl, with occasional replacement of alanyl by seryl units. With such an arrangement it is impossible for a single polypeptide chain to possess a twofold screw axis, and it must therefore be inferred that each twofold screw axis involves at least two separate chains.

\section{Arrangement of the polypeptide chains}

Before considering possible arrangements of the molecular chains in the unit cell it is necessary to discuss the form of the individual chains. Until recently it was generally accepted that the polypeptide chains in silk were in the fully extended form. However, it has been shown by Corey \& Donohue (1950) that this form requires a repeat distance of $7 \cdot 27 \AA$, whereas the fibre period in silk is $6.95 \AA$. It has therefore become clear

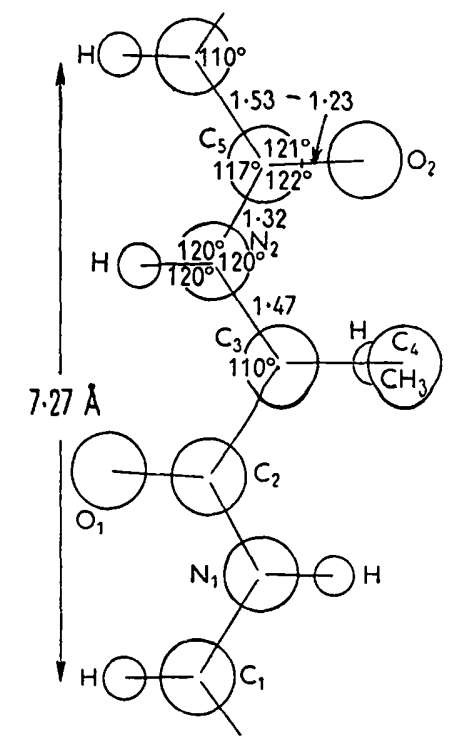

Fig. 3. Dimensions of the fully extended polypeptide chain (Corey \& Donohue). that the polypeptide chains in the crystalline regions of silk cannot be fully extended.

A study of possible configurations of the polypeptide chain was made with the help of an accurately constructed molecular model. The fully extended form of the chain was modified by rotations around bonds to the $\alpha$ carbon atoms (Pauling, Corey \& Branson, 1951), the amide group being kept planar. It was found that a small rotation about the $\mathrm{C}_{2}-\mathrm{C}_{3}$ bond (cf. Fig. 3 ) caused the $\mathrm{CH}_{3}$ group of the alanine residue to stand almost at right angles to the plane of the adjacent amide group, and that a small rotation about the $\mathrm{C}_{3}-\mathrm{N}_{2}$ bond made the $\mathrm{CO}$ groups point in parallel but opposite directions. These operations caused the chain to bend in such a manner that the repeat distance along it was $6.95 \AA$. Fig. 4 shows the resulting atomic

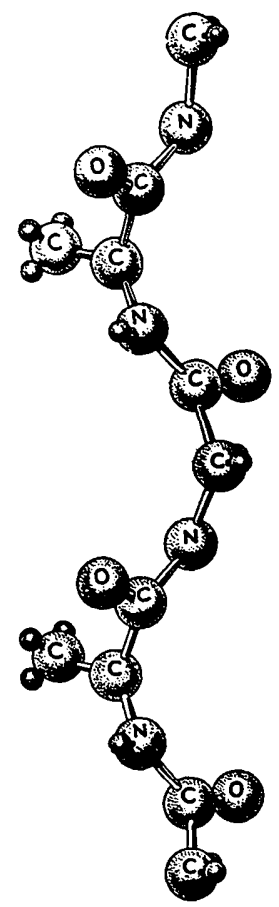

Fig. 4. Perspective drawing of modified polypeptide chain.

arrangement, which resembles the unit used by Pauling $\&$ Corey (1953) in the formulation of their pleatedsheet structure for $\beta$-type proteins and thought by them to be plausible for silk fibroin.

If the first unit cell of Kratky \& Kuriyama (1931) is accepted provisionally, the simplest space group consistent with the evidence is $P 2_{1}$, with two asymmetric units per cell. The polypeptide chains, bent in the manner discussed above, must then be arranged so that each chain is related to its neighbour by a twofold screw axis, and this arrangement can be easily achieved, with a reasonable hydrogen-bond distance of $2.85 \AA$ and a distance of $4.6 \AA$ between chain axes. As is shown diagrammatically in Fig. 5, such an arrangement fits the diagonal of the chosen cell and conforms to the space group $P 2_{1}$. 


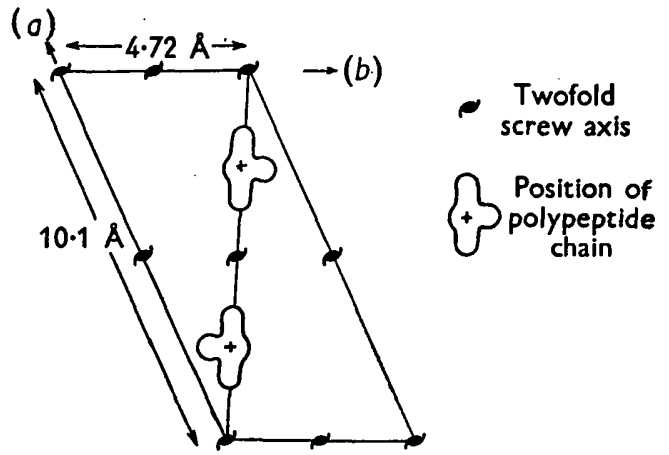

Fig. 5. Unit cell and possible arrangement of chains in conformity with space group $P 2_{1}$.

At first sight this structure appears to be a satisfactory solution to the problem, but difficulties arise when it is considered in relation to evidence provided by the work of Kratky \& Kuriyama (1931) with oriented silk films. These workers found that when the $\mathrm{X}$-ray beam was perpendicular to the direction of stretching but parallel to the film surface the diagram showed spots $A_{1}, A_{2}$, and $A_{4}$ on the equator; a photograph taken with the $\mathrm{X}$-ray beam at right angles to the surface of the film showed only the spot $A_{3}$ on the equator. The structure outlined above would require the (110) planes to be parallel to the film surface in these experiments if it is assumed that rolling or stretching orients the sheets of polypeptide chains parallel to this surface. With this orientation, consideration of the conditions of reflexion shows that the proposed structure would lead to results exactly the reverse of those obtained by Kratky \& Kuriyama. Furthermore, the most diffuse reflexions would be expected to be 110 and 220 , i.e. the part $A_{3}$ of the composite spot $\left(A_{2}+A_{3}\right)$ and the spot $A_{5}$, because the weakest forces of the structure are perpendicular to these planes; similar arguments show that the 100 , 200 and 300 reflexions, i.e. the spot $A_{1}$, the part $A_{2}$ of the composite spot $\left(A_{2}+A_{3}\right)$, and the spot $A_{4}$, should be sharp. Again the predicted results are the reverse of those found experimentally, particularly for the reflexions $A_{1}, A_{4}$ and $A_{5}$. Thus these considerations strongly suggest that the molecular chains are approximately at right angles to the chosen position.

If the molecular chains are turned through $90^{\circ}$ the plane of the sheets of chains becomes parallel to the $b$ axis, with the hydrogen bonding also in this direction. Since alternate chains are not equivalent, this change necessitates the doubling of the $b$ dimension of the unit cell, and in consequence the number of asymmetric units per cell becomes four, and the space group $P 2_{1}$ is no longer valid. The conditions of reflexion now become $h k l$ for $k \doteq 2 n, h k 0$ for $k=2 n$, and $00 l$ for $l=2 n$. The first condition is not consistent with any monoclinic space group, so that the most probable space group indicated is $P 2_{1} / b$, where $h k l$ appears for all orders. In the polypeptide chain only the residues of one of the two optical isomers of alanine are present so that there cannot be any planes of symmetry in the structure. Thus no arrangement of polypeptide chains with a glycyl-alanyl repeating unit can be formed with a glide plane of symmetry involving a mirror plane perpendicular to the axis of the chains, and hence it is impossible to fit such polypeptide chains into the space group $P 2_{1} / b$. No other monoclinic space group involving at least one twofold screw axis and having a cell with four asymmetric units could be found to fit the available evidence, and it was therefore necessary to seek another solution.

\section{Proposed structure for silk fibroin}

A new unit cell with $\gamma=86^{\circ}$ can be formed from the (100) and (110) planes of the Kratky-Kuriyama cell as shown in Fig. 6. When it is borne in mind that the dimensions of the original cell depend on measurements made on fibre diagrams, and hence are subject to considerable error, it is not unreasonable to take the angle of the derived cell as $90^{\circ}$. This immediately suggests orthorhombic symmetry for the crystallites of silk fibroin, a possibility noted by Kratky \& Kuriyama (1931) but not discussed in detail.

The orthorhombic unit cell proposed has the following dimensions:

$$
a=9 \cdot 29, b=9 \cdot 44, c=6.95 \AA ; z=4 .
$$

(a)

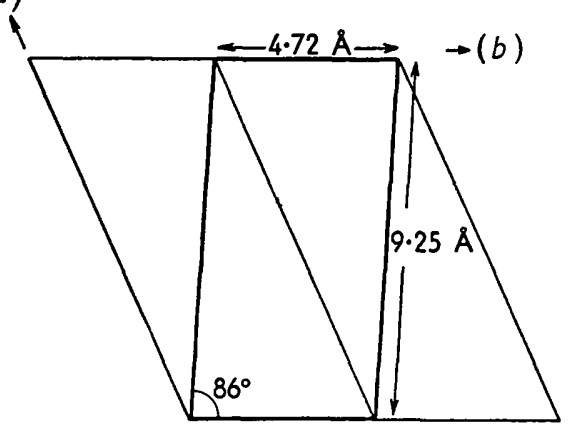

Fig. 6. Derivation of proposed unit cell.

The theoretical density of this cell is virtually the same as that of the Kratky-Kuriyama cell from which it is derived, and hence consistent with the evidence given earlier. All the reflexions can be indexed on the basis of the new cell, as is shown by Table 2 , and the conditions of reflexion are $h k l$ for all orders, $0 k 0$ for $k=2 n$, and $00 l$ for $l=2 n$. The suggested space group is thus $P 22_{1} 2_{1}$, which requires four asymmetric units in general positions.

Pauling \& Corey (1953) have suggested that a pleated sheet of polypeptide chains in antiparallel arrangement is possible for silk fibroin. Such a pleated sheet, formed with polypeptide chains having the configuration discussed above, can easily be fitted into the proposed cell if it is placed perpendicular to 
Table 2. Observed principal reflexions in fibre diagrams of silk fibroin

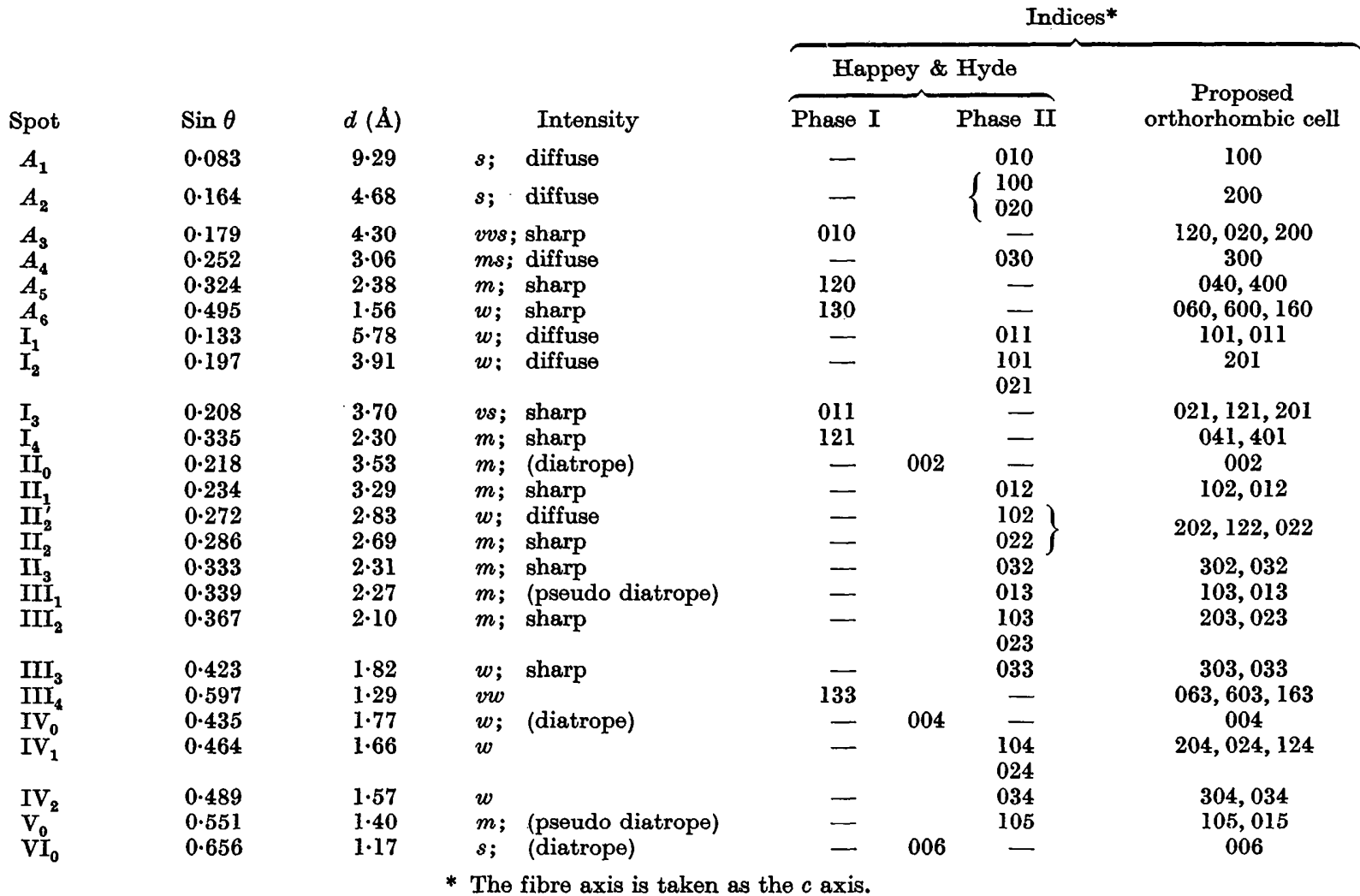

the $a$ axis. If a hydrogen-bond length of $2 \cdot 80-2 \cdot 85 \AA$ is assumed, the distance between chain axes is $4.72 \AA$, so that two chains of the sheet fit in the $b$ dimension of the unit cell. The repeat distance along the chains coincides with the $c$ dimension of the cell. If a second sheet is placed in the cell so that it is related to the first by a twofold screw axis parallel to the fibre axis, another screw axis parallel to the $b$ axis is also generated. It will be shown later that this can be done, with reasonable van der Waals distances between successive sheets, so as to give the $a$ dimension of the proposed unit cell. This arrangement thus fits into the proposed cell; it also conforms with the space group $P 22_{1} 2_{1}$ because it possesses the required twofold axis parallel to the $a$ axis and twofold screw axes parallel to both the $b$ and $c$ axes. These arguments will be clarified by reference to Fig. 7, which shows three views of the proposed structure.

This structure is consistent with the evidence of Kratky \& Kuriyama (1931) if the reasonable assumption is made that in a highly oriented fibroin film the pleated sheets, i.e. the (100) planes, lie parallel to the surface of the film. On the assumption that the dispersion of planes is $15-20^{\circ}$, as expected by Kratky \& Kuriyama, a photograph taken with the $\mathrm{X}$-ray beam parallel to the film surface should show the reflexions $A_{1}, A_{2}$ and $A_{4}$, indexed as 100,200 and 300 respectively, but not the reflexion $A_{3}$, indexed as 020 and 120 .
A photograph taken with the beam at right angles to the film surface could show the $010,020,030$ and 120 reflexions, assuming the same degree of dispersion. The 020 and 120 reflexions have very similar Bragg angles, and they appear as the spot $A_{3}$. The fact that the 010 and 030 reflexions do not appear is in full accord with the proposed space group. Kratky \& Kuriyama expected the planes contributing to the spot $A_{3}$ to be inclined at an angle of at least $40^{\circ}$ to the planes giving the reflexions $A_{1}, A_{2}$ and $A_{4}$; consideration of the unit cell shows that the (120) planes make an angle of $63^{\circ}$, and the (020) planes an angle of $90^{\circ}$, with the $(100)$ planes.

The proposed structure is also in agreement with the evidence advanced by Brill (1942). His unit cell* can be modified in a manner similar to that described above so as to give a cell with only slight dimensional differences from that proposed here. Moreover, Brill's arrangement of the molecular chains bears a general resemblance to that given above, so that it would be expected that the analogies between silk and synthetic polyamides which Brill noted should be preserved in the new structure.

A final test of the structure was made by comparing calculated and observed intensities. For the purpose

* It should be noted that the $a$ direction in Brill's unit cell corresponds to the $b$ direction in the proposed cell. 


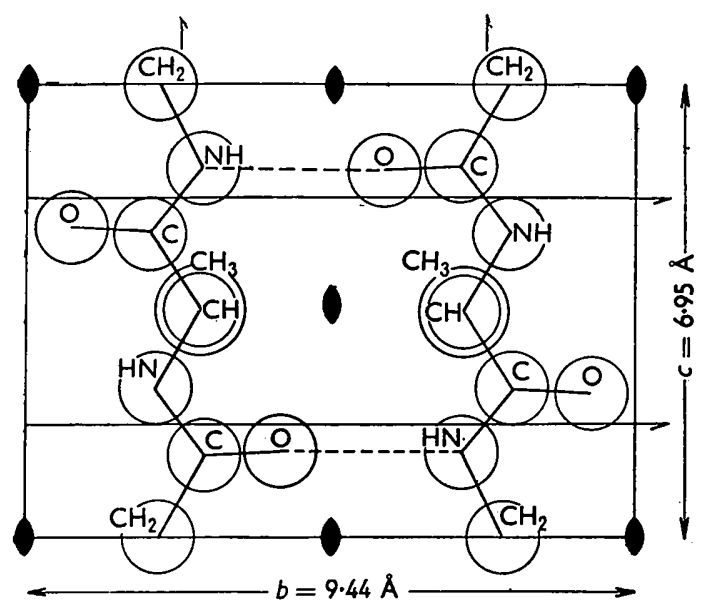

(a)

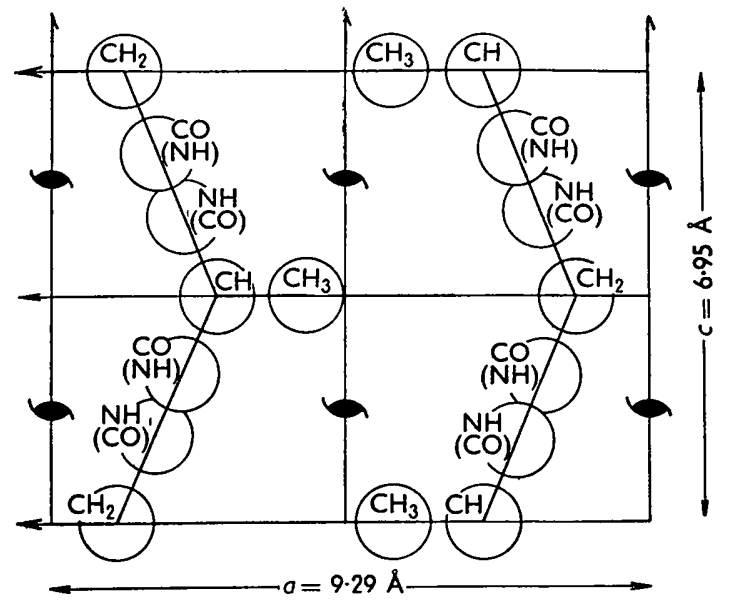

(b)

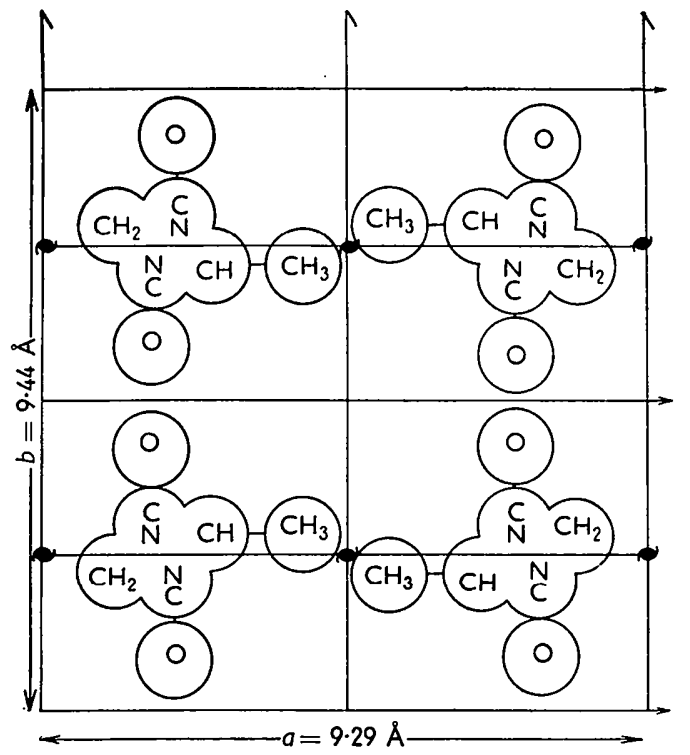

(c)

Fig. 7. Projections of proposed structure $(a)$ on (100), (b) on (010), (c) on (001).

of the calculation of the intensities it was necessary to assume that the polypeptide chains consisted of alternate glycine and alanine residues, the effect of the replacement of some of the alanine residues by serine being neglected. The effect of any distortion of the crystal lattice had also to be left out of account.

The co-ordinates of the various atoms in the structure were obtained by placing an accurate model of the molecular chain in parallel light, plotting two projections at right angles to each other, and then deriving the required co-ordinates from the two projections. A check of the accuracy of these co-ordinates was made by using them to calculate the bond lengths between atoms and comparing the values obtained with those used in the model. An overall mean error of approximately $1 \%$ was found, and this was considered to be well within the accuracy of the X-ray data. The chain was placed in the unit cell with its axis at $x=0.2$ and $y=0.25$ and the $\mathrm{CH}_{2}$ group at $z=0$. This position permits the symmetry operations required by the space group $P 22_{1} 2_{1}$, and each sheet fits into the folds of the preceding sheet with values of 4.0 and $3.7 \AA$ for the distances

$$
>\mathrm{CH} . \mathrm{CH}_{3} \ldots>\mathrm{CH}_{2} \text { and }>\mathrm{CH}_{2} \ldots>\mathrm{CH} . \mathrm{CH}_{3}
$$

respectively; these values are reasonable for the operation of van der Waals forces. The atomic co-ordinates are given in Table 3.

The structure factors for all the principal planes contributing to the normal fibre diagram were computed from the appropriate equations for the space group $P 22_{1} 2_{1}$ (International Tables, 1952). The atomic 
Table 3. Co-ordinates of atoms in proposed orthorhombic cell

$\begin{array}{cccc}\text { Atom } & X / a & Y / b & Z / c \\ \mathrm{C}_{1} & 0.12 & 0.22 & 0 \\ \mathrm{C}_{2} & 0.22 & 0.20 & 0.33 \\ \mathrm{C}_{3} & 0.28 & 0.28 & 0.50 \\ \mathrm{C}_{4} & 0.44 & 0.28 & 0.51 \\ \mathrm{C}_{5} & 0.18 & 0.29 & 0.82 \\ \mathrm{C}_{6} & 0.12 & 0.22 & 1.00 \\ \mathrm{O}_{1} & 0.22 & & \\ \mathrm{O}_{2} & 0.18 & 0.42 & 0.32 \\ \mathrm{~N}_{1} & 0.18 & 0.29 & 0.81 \\ \mathrm{~N}_{2} & 0.22 & 0.21 & 0.18 \\ & & & 0.68\end{array}$

scattering factors used in these calculations were taken from graphs drawn from the data for carbon, nitrogen and oxygen in Internationale Tabellen (1935), but no temperature correction was applied. The relative intensities of reflexions were calculated from the formula

$$
I \propto L F^{2} / D_{1},
$$

where $F$ is the structure factor, $L$, given by the expression $\left(1+\cos ^{2} 2 \theta\right) / \sin 2 \theta$, is the combined Lorentz and polarization factor, and $D_{1}$, given by the expression $V\left\{\cos ^{2} \varphi-\sin ^{2} \theta\right\} / \mathrm{cos} \theta$, is the geometrical cor-

Table 4. Calculated and observed intensities of reflexions in fibre diagrams of silk fibroin

(a) Fibre bundle vertical

\begin{tabular}{|c|c|c|c|c|}
\hline \multirow[b]{2}{*}{ Spot } & \multirow{2}{*}{$\begin{array}{l}\text { Reflexions } \\
\text { included }\end{array}$} & \multicolumn{3}{|c|}{ Relative intensity } \\
\hline & & Obs. & & alc. \\
\hline $\begin{array}{l}A_{1} \\
A_{2} \\
A_{3} \\
A_{4} \\
A_{5} \\
A_{8}\end{array}$ & $\begin{array}{c}100 \\
200 \\
020,120 \\
300 \\
040,400 \\
600,060,160\end{array}$ & $\left.\begin{array}{l}s ; \text { diffuse } \\
s ; \text { diffuse } \\
v v s ; \text { sharp } \\
m-s ; \text { diffuse } \\
m ; \text { sharp } \\
w ; \text { sharp }\end{array}\right\}$ & $\begin{array}{r}370 \\
1335 \\
396 \\
64 \\
38\end{array}$ & $\begin{array}{l}s \\
v v s \\
s \\
m \\
w-m\end{array}$ \\
\hline $\begin{array}{l}I_{1} \\
I_{2} \\
I_{3} \\
I_{4}\end{array}$ & $\begin{array}{c}101,011 \\
201,211 \\
021,121 \\
041,401,321,231,141\end{array}$ & $\left.\begin{array}{l}w \text {; diffuse } \\
w ; \text { diffuse } \\
v s ; \text { sharp } \\
m ; \text { sharp }\end{array}\right\}$ & $\begin{array}{r}33 \\
376 \\
93\end{array}$ & $\begin{array}{l}w-m \\
s \\
m\end{array}$ \\
\hline $\begin{array}{l}\mathrm{II}_{0} \\
\mathrm{II}_{1}\end{array}$ & $\begin{array}{c}002 \\
102,012\end{array}$ & $\begin{array}{l}m \\
m ; \text { sharp }\end{array}$ & $\begin{array}{l}12 \\
63\end{array}$ & $\begin{array}{l}w \\
m\end{array}$ \\
\hline $\mathrm{II}_{2}$ & $202,122,022$ & $m\left\{\begin{array}{l}\text { diffuse edge } \\
\text { to spot }\end{array}\right.$ & 184 & $s$ \\
\hline $\mathrm{II}_{3}$ & $302,032,132$ & $m ;$ sharp & 71 & $m$ \\
\hline $\mathrm{III}_{1}$ & 103,013 & $m$ & 493 & vs \\
\hline $\begin{array}{l}\mathrm{III}_{2} \\
\mathrm{III}_{3} \\
\mathrm{III}_{4}\end{array}$ & $\begin{array}{l}203,023,213 \\
303,033,313 \\
063,603,163\end{array}$ & $\begin{array}{l}m ; \text { sharp } \\
w ; \text { sharp } \\
v w ; \text { diffuse }\end{array}$ & $\begin{array}{r}99 \\
111 \\
5\end{array}$ & $\begin{array}{l}m \\
m-s \\
v w\end{array}$ \\
\hline
\end{tabular}

(b) Fibre bundle horizontal $00 l$ Reflexions

\begin{tabular}{ccccc} 
& \multicolumn{4}{c}{$00 l$ Reflexions } \\
Spot & Reflexion & Obs. & Calc. \\
$\mathrm{II}_{0}$ & 002 & $m$ & 12 & $w$ \\
$\mathrm{IV}_{0}$ & 004 & $w$ & 10 & $w$ \\
$\mathrm{VI}_{0}$ & 006 & $s$ & 57 & $m$
\end{tabular}

rection factor of Cox \& Shaw (1930) which allows for the inclination of the reflecting planes, $\varphi$ being the angle between the plane and the axis of rotation. No allowance was made for film absorption since appropriate data were not available, but the error produced by neglecting it is expected to be very small. The results of these calculations are compared with the observed intensities in Table 4(a).

Some discussion of the intensities of the reflexions $A_{2}$ and $A_{3}$ is necessary. It is found that the Bragg angles of the 200,020 and 120 reflexions are so similar that the reflexions will overlap in a fibre photograph, producing one very intense spot identified as $A_{3}$. However, some distortion of the lattice in a direction perpendicular to the (100) planes is to be expected, and this will have the effect of rendering the reflexions from these planes broad and diffuse. Thus the secondorder reflexion from the (100) planes, i.e. the reflexion indexed as 200, appears as a fringe to the reflexion $A_{3}$ and is identified as $A_{2}$. For this reason the reflexions $A_{2}$ and $A_{3}$ have been treated as a single spot in the calculations. Similar arguments apply to $I_{2}$ and $I_{3}$. When these considerations are taken into account, the agreement between the observed and calculated intensities may be regarded as satisfactory for the equatorial reflexions and fairly good for the other reflexions.

Similar calculations were made for the $00 l$ reflexions found on the equator of the photographs taken with a horizontal bundle of fibres. Table $4(b)$ shows that here also the agreement between observed and calculated intensities is as good as could be expected.

Calculations of intensities were also made for about a hundred reflexions which theoretically could occur but are not visible in the fibre photographs. The calculated intensity, expressed as a percentage of that of the strongest observed reflexion, was $1-5 \%$ for nine reflexions, viz. 500, 501, 502, 402, 301, 241, 053, 221 and 232 , and less than $1 \%$ for all the rest. In view of the fact that the calculations take no account of lattice distortion and the presence of serine residues in the polypeptide chains, these results are not inconsistent with the validity of the structure.

The proposed structure thus provides a reasonably satisfactory explanation not only of the positions but also of the intensities of the $\mathrm{X}$-ray reflexions from silk fibroin, and it may therefore be regarded as at least an approximation to the structure of the crystalline portions of the silk fibre.

\section{Experimental}

\section{Determination of density}

The density of the insoluble fraction obtained by the action of pancreatic enzymes on silk fibroin was determined in benzene at $20^{\circ} \mathrm{C}$. by means of a specificgravity bottle. The measurement was made on material that had been dried over phosphorus pentoxide. 


\section{$X$-ray photographs}

The X-ray photographs were taken with nickelfiltered copper radiation from a tube working at 45 $\mathrm{kVp}$. and $15 \mathrm{~mA}$., and recorded on Ilford Industrial B X-ray film. The collimator consisted of a lead-glass capillary tube, the bore of which was $0.25 \mathrm{~mm}$. for the powder photographs and $0.5 \mathrm{~mm}$. for the fibre photographs.

For the powder photographs a sample of the material weighing $20 \mathrm{mg}$. was compressed into a pellet and mounted on the goniometer head of a $3 \mathrm{~cm}$. cylindrical camera; the specimen was kept stationary during the exposure. Confirmatory photographs were taken with cylindrical specimens, $1 \mathrm{~mm}$. in diameter, which were obtained by moulding the powdery materials after they had been moistened with a $0.5 \%$ aqueous solution of gum arabic; in these cases the specimens were rotated. The time of exposure was $3 \mathrm{hr}$. for all the materials examined.

The normal fibre photograph was taken with a vertical bundle of degummed silk fibres kept under tension by means of a special holder adapted for use with the $3 \mathrm{~cm}$. cylindrical camera; the diameter of the bundle was about $1 \mathrm{~mm}$. After satisfactory photographs had been obtained the fibre bundle was moistened with a $2 \%$ aqueous solution of gum tragacanth and allowed to dry while still under tension. A new photograph showed that this treatment caused no detectable change in the $\mathrm{X}$-ray diagram. A short portion was then cut from this fibre bundle with a sharp razor-blade, and it was attached to the end of a glass fibre and mounted on the goniometer head of the cylindrical camera with the fibre axis horizontal; the method of mounting and adjustment was essentially that used for single crystals. Oscillation photographs were then taken at various settings of the fibre bundle relative to the $\mathrm{X}$-ray beam, the amplitude of the oscillation being $15^{\circ}$. An exposure of $1-1.5 \mathrm{hr}$. was required.

\section{References}

BRmL, R. (1923). Liebigs Ann. 434, 204.

BrILL, R. (1942). Z. phys. Chem. B, 53, 61.

Corey, R. B. \& Donohue, J. (1950). J. Amer. Chem. Soc. 72, 2899.

Cox, E. G. \& Shaw, W. F. B. (1930). Proc. Roy. Soc. A, $127,71$.

Drucker, B., Hainsworth, R. \& Smith, S. G. (1953a). Shirley Inst. Mem. 26, 191.

DrdCker, B., Hainsworth, R. \& SMrth, S. G. (1953b). J. Text. Inst., Manchr. 44, T420.

Drucker, B. \& Smith, S. G. (1950). Nature, Lond. 165, 196.

Goldschmidt, S. \& Strauss, K. (1930). Liebigs Ann. $480,263$.

Goodings, A. C. \& TuRL, L. H. (1940). J. Text. Inst., Manchr. 31, T69.

HaPPey, F. \& HYDE, A. J. (1952). Nature, Lond. 169, 921.

Herzog, R. O. \& JANCKE, W. (1920). Ber. dtsch, chem. Ges. 53, 2162.

International Tables for X-ray Crystallography (1952). Birmingham: Kynoch Press.

Internationale Tabellen zur Bestimmung von Kristallstrukturen (1935). Vol. 2. Berlin: Borntraeger.

KRATKY, O. (1929). Z. phys. Chem. B, 5, 297.

Kratky, O. \& Kuriyama, S. (1931). Z. phys. Chem. B, 11, 363.

Legrand, C. (1952). Acta Cryst. 5, 800.

Levy, M. \& Slobodian, E. (1952). J. Biol. Chem. 199, 563.

MeYeR, K. H., Fuld, M. \& Klemm, O. (1940). Helv. chim. Acta, 23, 1441.

Meyer, K. H. \& Mark, H. (1928). Ber. dtsch. chem. Ges. 61, 1932.

Pauling, L. \& Corey, R. B. (1953). Proc. Roy. Soc. B, 141, 21.

Padling, L., Corey, R. B. \& Branson, H. R. (1951). Proc. Nat. Acad. Sci., Wash. 37, 205.

Slobodian, E. \& Levy, M. (1953). J. Biol. Chem. 201, 371.

Trogus, C. \& Hess, K. (1933). Biochem. Z. $260,376$. 